The algebraic geometry of perfect and sequential equilibrium: an extension

Xiao Luo, Xuewen Qian, Yang Sun

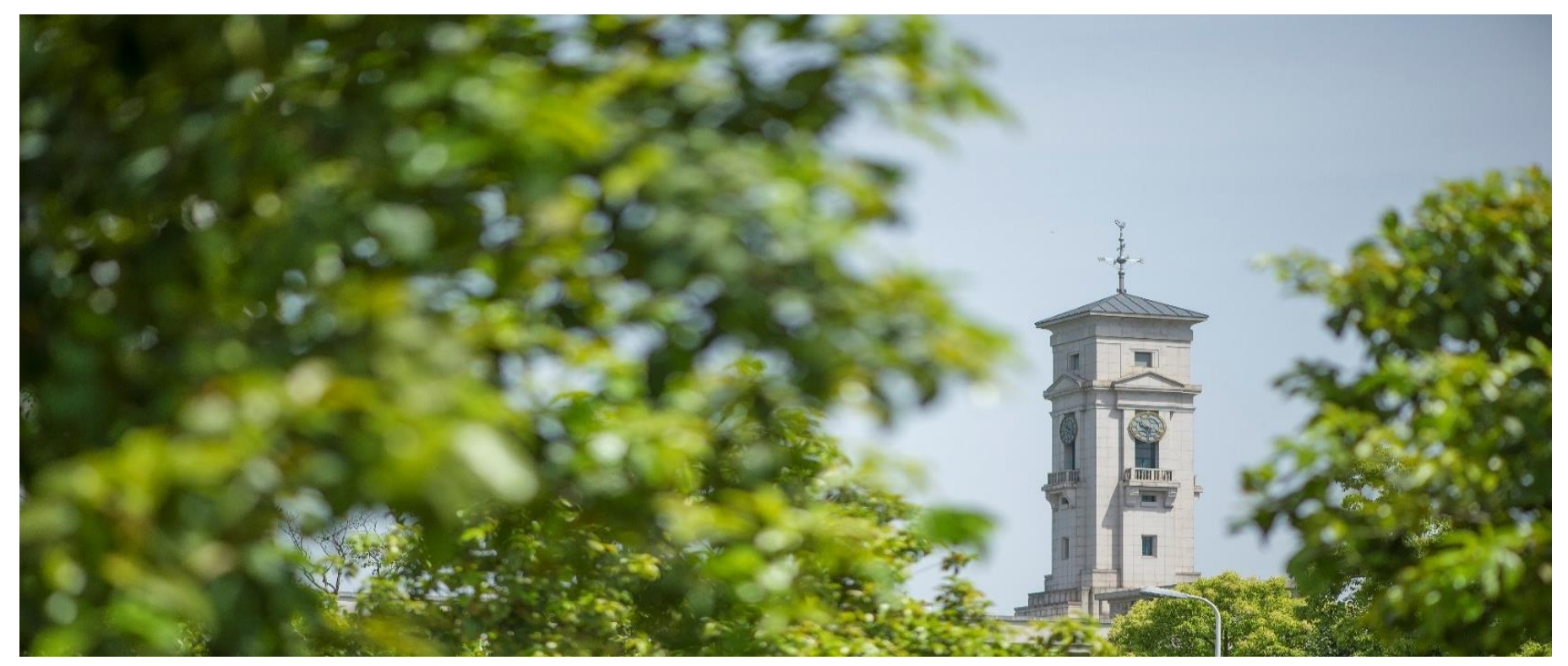


Faculty of Humanities and Social Science, University of Nottingham Ningbo China, 199 Taikang East Road, Ningbo, 315100, Zhejiang, China.

First published 2020

This work is made available under the terms of the Creative Commons Attribution 4.0 International License:

http://creativecommons.org/licenses/by/4.0

The work is licenced to the University of Nottingham Ningbo China under the Global University Publication Licence:

https://www.nottingham.edu.cn/en/library/documents/researchsupport/global-university-publications-licence.pdf 


\title{
The algebraic geometry of perfect and sequential equilibrium: an extension*
}

\author{
Xiao Luo ${ }^{\mathrm{a}}$, Xuewen Qian ${ }^{\mathrm{b}}$, Yang $\mathrm{Sun}^{\mathrm{c} \dagger}$ \\ ${ }^{a}$ Department of Economics, National University of Singapore, Singapore 117570 \\ ${ }^{\mathrm{b}}$ School of Economics, University of Nottingham Ningbo China, Ningbo 315100 \\ ${ }^{\mathrm{c}}$ School of Economics, Sichuan University, China 610065
}

\begin{abstract}
We extend the generic equivalence result of Blume and Zame (Econometrica 62: 783-794, 1994) to a broader context of perfectly and sequentially rational strategic behavior (including equilibrium and nonequilibrium behavior) through a unifying solution concept of "mutually acceptable course of action" (MACA) proposed by Greenberg et al. (2009). As a by-product, we show, in the affirmative, Dekel et al.'s (1999) conjecture on the generic equivalence between the sequential and perfect versions of rationalizable self-confirming equilibrium. JEL Classification: C70, C72
\end{abstract}

Keywords: Extensive forms; generic payoffs; perfect rationality; sequential rationality; MACA; semi-algebraic sets

\footnotetext{
${ }^{*}$ We are grateful to the Editor, an Associate Editor, and two anonymous referees for valuable comments. We thank Yi-Chun Chen, Shravan Luckraz, Yongchuan Qiao, Yeneng Sun and Satoru Takahashi for helpful comments and discussions. An earlier version of the paper was presented at the Asian Meeting of the Econometric Society, Singapore; and the SAET Conference in Faro, Portugal. Financial support from the National University of Singapore is gratefully acknowledged. The usual disclaimer applies.

${ }^{\dagger}$ Corresponding author. E-mail: ecslx@nus.edu.sg (X. Luo), Xuewen.Qian@nottingham.edu.cn (X. Qian), sunyang789987@gmail.com (Y. Sun).
} 


\title{
1 Introduction
}

In dealing with the imperfection in extensive-form games (with perfect recall), Selten (1975) introduced the notion of perfect equilibrium. A perfect equilibrium is a Nash equilibrium that takes the possibility of off-the-equilibrium play into account by assuming that the players, through the idea of "trembling hand," may choose all unintended strategies, albeit with small probabilities. In the spirit of Selten's (1975) perfectness, Kreps and Wilson (1982) proposed the notion of sequential equilibrium, by imposing the so-called "sequential consistency" and "sequential rationality" on the behavior of every player. Sequential equilibrium is more inclusive and weaker than perfect equilibrium: Every perfect equilibrium must be a sequential equilibrium strategy profile. Kreps and Wilson (1982) pointed out that the two concepts lead to similar prescriptions for equilibrium play-that is, for each particular game form and for almost all assignments of payoffs to the terminal nodes, almost all sequential equilibria are perfect equilibria, and the sets of sequential and perfect equilibria fail to coincide only at payoffs where the perfect equilibrium correspondence fails to be upper hemi-continuous. Blume and Zame (1994) (hereafter BZ94) strengthened Kreps and Wilson's (1982) result, and showed that for almost all assignments of payoffs to the terminal nodes, the sets of sequential and perfect equilibria are identical.

BZ94 obtained the generic equivalence result by exploiting the semi-algebraic structure of the graphs of the perfect and sequential equilibrium correspondences (because the graphs of these correspondences can each be written as a subset of a Euclidean space defined by a finite number of polynomial equalities and inequalities) ${ }^{1}$ As they pointed out,

\begin{abstract}
We believe that, just as differential topology has proved to be the right tool for studying the fine structure of the Walrasian equilibrium correspondence, so will real algebraic geometry prove to be the right tool for studying the fine structure of game-theoretic equilibrium correspondences. (BZ94, p.784)
\end{abstract}

In this paper, we follow BZ94 to further study the relationship between perfectly and sequentially rational strategic behavior in a broad sense, including equilibrium and non-equilibrium solution concepts (e.g., rationalizability and self-confirming equilibrium), from the point of view of semi-algebraic geometry.

By using Greenberg et al.'s (2009) concept of "mutually acceptable courses of action" (MACA), we obtain a unified generic equivalence in a broader context of perfectly and sequentially rational strategic behavior (Theorem 1). More specifically, we establish a general generic equivalence between the sequential and perfect notions of MACA with an elaborate belief structure in an extensive-form game, namely the "extensive-form convex hull" introduced by Dekel et al. (2002). The notion of

\footnotetext{
${ }^{1}$ By the Tarski-Seidenberg Theorem, many equilibrium solution concepts can be defined by a first-order formula and thus the equilibrium correspondences are semi-algebraic (cf. BZ94, Theorems 1 and 2). A semi-algebraic set has the following structure: Each semi-algebraic set has only a finite number of open connected components, and has a welldefined dimension (see, e.g., Hardt (1980) and Bochnak et al. (1987)).
} 
extensive-form convex hull models the subjective uncertainty of a player over the behavioral strategies of an opponent especially in the non-equilibrium paradigm, and it essentially restricts the scope of the limit points of trembling sequences in the analysis of perfectly and sequentially rational behavior in extensive-form games ${ }^{2}$ We apply our generic equivalence to various game-theoretic solution concepts such as equilibrium, self-confirming equilibrium, and rationalizability, and obtain a variety of generic equivalence results as corollaries of our approach (Corollaries 1-3). In particular, Corollary 2(iii) shows, in the affirmative, Dekel et al.'s (1999) conjecture that the sequentially and perfectly rationalizable self-confirming equilibria are generically equivalent ${ }^{3}$

From a technical perspective, BZ94's approach relies heavily on the semi-algebraic property of sets of perfect/sequential equilibria, which are defined by polynomial equalities and inequalities in finite dimensional Euclidean spaces. More specifically, BZ94 obtained generic equivalence between perfect and sequential equilibria, based on the fact that the graphs of the perfect and sequential equilibrium correspondences can be characterized respectively by the closure and vertical closure of the (semialgebraic) graph of the perturbed Nash equilibrium correspondence. However, it is less clear that other kinds of game-theoretic solution concepts -such as perfect and sequential versions of self-confirming rationalizable equilibrium and rationalizability defined in Dekel et al. (1999, 2002) and Greenberg et al. (2009) - have the semi-algebraic property, because the definitions involve a complex structure of beliefs in terms of the extensive-form convex hull. Rather than working directly on the semi-algebraic property of specific solution concepts, we here take an alternative approach based on the semi-algebraic property of the primitive set of "perfectly-rational states." A state of a player consists of three elements: the player's payoff function, his belief over opponents' choice and his strategy. A perfectly-rational state is a state with the restriction that the player's choice of strategy is optimal with respect to his payoff function and "cautious" belief (in terms of a full-support behavior strategy profile). Our general generic equivalence result follows from, in the spirit of Kreps and Wilson (1982, Proposition 6 ), a characterization of sequential/perfect rationality by the closure and vertical closure of the set of perfectly-rational states, with the additional restrictions that the limit point of a sequence of trembles is confined to the extensive-form convex hull.

Our approach is feasible and applicable to a variety of situations, as long as the sequence of trembles in the analysis of perfectly and sequentially rational behavior is concordant with the diverse belief structure adopted in an extensive-form game. For instance, if the belief structure is restricted to a "common" sequence of trembles for all players, we obtain BZ94's generic equivalence result for perfect and sequential equilibria (Corollary 1); if the belief structure allows different players to have

\footnotetext{
${ }^{2}$ The notion of extensive-form convex hull is designed to overcome the notorious problem of imperfection under subjective uncertainty over (behavioral) strategies in extensive-form games. Two important features of making use of the notion of the extensive-form convex hull are (i) it eliminates weakly dominated strategies in simultaneous-move games, and (ii) it yields the backward-induction strategy profile in a "generic" perfect-information game.

${ }^{3}$ Dekel et al. (1999, Footnote 4) made a claim without proof: "There are two closely related notions of optimality at offpath information sets that we consider: best replies to the limit of a sequence of trembles, namely sequential rationality, as in Kreps and Wilson (1982), and best replies to the sequence itself, as in Selten's notion (1975) of trembling-hand perfection. We expect that, as in the relationship between sequential and perfect equilibrium, the difference is only in non-generic games see Kreps and Wilson (1982) and Blume and Zame (1994) but verifying this takes us too far afield."
} 
distinct sequences of trembles $4^{4}$ we obtain a generic equivalence result for "weakly" perfect and "weakly" sequential equilibria (Corollary 2(i)).

The research line of "genericity" in game theory sheds light on important and fundamental issues on rational strategic behavior 5 e.g., Kreps and Wilson (1982) and Kohlberg and Mertens (1986) applied Sard's Theorem and the Regular Value Theorem in differential topology to study equilibrium distributions over terminal nodes and the generic finiteness of equilibria components (see also Blume and Zame (1993), Ritzberger (1994), McKelvey and McLennan (1996), Kohlberg and Reny (1997), Perea et al. (1997), Govindan and Wilson (2001, 2006, 2012), Govindan and McLennan (2001), Hillas and Kohlberg (2002), Haller and Lagunoff (2002), Demichelis and Ritzberger (2003), Voorneveld (2005), Kubler and Schmedders (2010), Pimienta and Litan (2008), and Pimienta and Shen (2014) for more discussions).

The rest of the paper is organized as follows. In Section 2, we provide an illustrative example to explain the general generic equivalence between perfectly and sequentially rational strategic behavior. In Section 3, we introduce the set-up, notation, and definitions. In Section 4, we present the central result on the general generic equivalence, and apply our approach to various game-theoretic solution concepts. Section 5 concludes. To facilitate reading, we relegate all the proofs to Appendix B and relevant definitions to Appendix A.

\section{An Illustrative Example}

In a "generic" game with perfect information (i.e., the game is not a "nongeneric" case where, for some player, the same payoff is assigned to two distinct terminal nodes), it is fairly easy to see that perfect/sequential equilibrium yields the unique backward-induction strategy profile. In other words, sequential and perfect equilibria are generically identical in games with perfect information $\sqrt[6]{6}$ A similar result indeed holds true for the notion of perfect/sequential rationalizability. Thus, in the class of "generic" games with perfect information (excluding a lower-dimensional set of payoffs), both perfect/sequential equilibrium and rationalizability lead to the unique backward-induction strategy profile.

The following two-person extensive-form game demonstrates a "generic" equivalence relationship between perfectly and sequentially rational strategic behavior in a broad sense.

Apparently, $L$ dominates $R$ (for player 1 ); $l$ dominates $r$ (for player 2 ) if payoffs $u \leq 1$. It is easy to see that sequential equilibrium differs from perfect equilibrium only at the "nongeneric" payoff $u^{0}=1$ :

\footnotetext{
${ }^{4}$ That is, different players are not required to hold the same beliefs on how players "tremble". Fudenberg and Tirole (1991, p.341) pointed out, "Why should all players have the same theory to explain deviations that, after all, are either probability-0 events or very unlikely, depending on one's methodological point of view? The standard defense is that this requirement is in the spirit of equilibrium analysis, since equilibrium supposes that all players have common beliefs about the others' strategies. Although this restriction is usually imposed, we are not sure that we find it convincing."

${ }^{5}$ See also Aumann (1985) and van Damme (1998) for Robert Aumann's insightful and thoughtful discussions on the importance of "relationships" in scientific research.

${ }^{6}$ More precisely, a statement is "generically" true if it is false only for a lower dimensional subset of the payoff vector space. Besides full dimension we used here, there are other notions of "genericity" such as open and dense, residual, meagre complement, almost surely, and almost everywhere.
} 

between perfect and sequential equilibria occurs only for "nongeneric" payoff(s) in a lower-dimensional payoffs space. BZ94 showed that for "almost all" or "generic" assignments of payoffs to the terminal nodes, the sets of sequential and perfect equilibria are identical 7

This generic equivalence result does not appear to hinge on the fact that the solution concept is equilibrium-based. This kind of generic equivalence relationship indeed holds true for perfectly and sequentially rational strategic behavior in a broad sense-that is, "sequential rationality" differs from "perfect rationality" only at the "nongeneric" payoff $u^{0}=1$. For simplicity, we restrict attention to player 2's strategic behavior in Figure 1. Obviously, strategy $r$ is not perfectly rational for player 2 since $l$ (weakly) dominates $r$ at $u^{0}=1$. But, $r$ is sequentially rational when $u^{0}=1$ if player 2 holds a belief assessment that assigns a probability of 1 on the right-side node at his information set; the assessment can be generated by a "trembling sequence" $x^{\varepsilon} \equiv \varepsilon^{2} L+\varepsilon R+\left(1-\varepsilon-\varepsilon^{2}\right) S$ as $\varepsilon \rightarrow 0$. However, $r$ can be perfectly rational by allowing for payoff perturbations. That is, although $r$ is not optimal along the trembling sequence $x^{\varepsilon}, r$ can be optimal along the trembling sequence $x^{\varepsilon}$ under a slightly perturbed payoff $u^{\varepsilon}=1+2 \varepsilon$. Subsequently, that strategy $r$ is sequentially rational can be obtained from a limit point of "perfectly-rational states" $\left(u^{\varepsilon}, x^{\varepsilon}, r\right)$, i.e., $\lim _{\varepsilon \rightarrow 0}\left(u^{\varepsilon}, x^{\varepsilon}, r\right)=(1,1 \circ S, r)$.

In general, every sequentially rational strategy can be characterized by a limit point of perfectlyrational states (cf. Kreps and Wilson (1982, Proposition 6)), while every perfectly rational strategy is naturally associated with a limit point of perfectly-rational states, without payoff perturbations. That is, the set of sequentially (resp. perfectly) rational strategies can be characterized by the closure (resp. vertical closure) of the set of perfectly-rational states. By Generic Local Triviality in semi-algebraic geometry, the closure and vertical closure of the set of perfectly rational states are almost the same. It

\footnotetext{
${ }^{7}$ This example shows that there is no "generic" equivalence between Myerson's (1978) proper equilibrium and perfect equilibrium: For "generic" payoffs $u>1,(S, r)$ is a perfect equilibrium but not a proper equilibrium. van Damme (1992, Theorem 2.6.1) presented an "almost all" theorem: In "almost all" normal form games, Nash equilibria are "regular" equilibria (hence proper equilibria). Nevertheless, as van Damme (1992, p.45) pointed out, the analysis "is of limited value for the study of extensive form games as any nontrivial such game gives rise to a nongeneric normal form."
} 
therefore follows that sequential rationality differs from perfect rationality only at "nongeneric" payoffs. By using Greenberg et al.'s (2009) notion of MACA, we can establish a unified generic equivalence result between perfectly and sequentially rational strategic behavior in a wide range of environments (Theorem $1)$.

\section{Preliminaries}

\subsection{Set-up}

We consider a (finite) extensive form with perfect recall $\mathrm{8}^{8}$

$$
\Gamma=\left(N, V, H,\left\{A_{h}\right\}_{h \in H}\right),
$$

where $N$ is the finite set of players, $V$ is the finite set of nodes, $H$ is the finite set of information sets, $A_{h}$ is the finite set of actions available at information set $h$. Let $Z \subseteq V$ denote the finite set of terminal nodes. A payoff function for player $i$ is a function $u_{i}: Z \mapsto \mathbb{R}$. Let $U=\Pi_{i \in N} U_{i}$ where $U_{i}=\mathbb{R}^{|Z|}$ is the space of player $i$ 's payoff functions. The game $\Gamma(u)$ is specified by the extensive form $\Gamma$ and the payoffs $u \in U$.

A mixed action at information set $h$ is a probability distribution over the actions in $A_{h}$. Let $\mathbb{Y}_{h}$ denote the set of mixed actions at $h$; that is, $\mathbb{Y}_{h}=\Delta\left(A_{h}\right)$. The set of player $i$ 's (behavioral) strategies is $\mathbb{Y}_{i}=\Pi_{h \in H_{i}} \mathbb{Y}_{h}$ (where $H_{i}$ is the set of player $i$ 's information sets); that is, a strategy $y_{i} \in \mathbb{Y}_{i}$ of player $i$ specifies for each information set $h \in H_{i}$ a probability distribution $y_{h}$ over the action set $A_{h}$. Let $\mathbb{Y}=\Pi_{i \in N} \mathbb{Y}_{i}$ and $\mathbb{Y}_{-i}=\Pi_{j \neq i} \mathbb{Y}_{j}$. For a profile $y \in \mathbb{Y}$, we also write $y=\left(y_{i}, y_{-i}\right)=\left(y_{h}, y_{-h}\right)$.

The sets $\mathbb{Y}, \mathbb{Y}_{i}, \mathbb{Y}_{-i}$ and $\mathbb{Y}_{-h}$ can be viewed as semi-algebraic sets, which are defined by linear equalities and inequalities, in finite dimensional Euclidean spaces $9^{9}$ Fix a terminal node $z$, the probability $\operatorname{Pr}(z \mid y)$ that $z$ is realized by $y$ (from the initial node) is a polynomial function of $y \in \mathbb{Y}$. In game $\Gamma(u), i$ 's expected payoff function $v_{i}(\cdot, \cdot)$ is defined as follows: For all $y \in \mathbb{Y}$ and $u_{i} \in U_{i}$,

$$
v_{i}\left(y, u_{i}\right)=\Sigma_{z \in Z} u_{i}(z) \operatorname{Pr}(z \mid y)
$$

which is semi-algebraic on $\mathbb{Y} \times U_{i}$. Let $\operatorname{int}(\mathbb{Y})$ denote the semi-algebraic set of strictly-positivebehavioral-strategy profiles in $\mathbb{Y}$-each of which assigns strictly positive probability to every action at every information set.

\footnotetext{
${ }^{8}$ Since the formal description of an extensive form is by now standard (see, for instance, Kreps and Wilson (1982) and Osborne and Rubinstein (1994)), we here include the necessary notation only. We note our approach can be extended to games with nature moves.

${ }^{9} \mathrm{~A}$ set $X \subseteq \mathbb{R}^{n}$ is semi-algebraic if it is the finite union of sets of the form $\left\{x \in \mathbb{R}^{n}: f_{1}(x)=0, \cdots, f_{k}(x)=0\right.$ and $\left.g_{1}(x)>0, \cdots, g_{m}(x)>0\right\}$, where the $f_{i}$ and $g_{j}$ are polynomials with real coefficients. A correspondence is semi-algebraic if and only if its graph is a semi-algebraic set.
} 


\subsection{Perfect rationality and sequential rationality}

Let $y_{i}^{t} \rightsquigarrow y_{i}$ denote a trembling sequence $\left\{y_{i}^{t}\right\}_{t=0}^{\infty}$ of strictly positive behavioral strategies in $\mathbb{Y}_{i}$ that converges to $y_{i}$ in $\mathbb{Y}_{i}$ as $t$ goes to infinity. Let $y^{t} \rightsquigarrow y$ denote $y_{i}^{t} \rightsquigarrow y_{i} \forall i \in N$. (Throughout this paper, we consider the pointwise convergence in a Euclidean space.) A consistent assessment is a profile-anddistributions pair $(y, \mu)$, where $y$ is a profile of behavioral strategies and $\mu$ is a function that assigns to every information set a probability distribution on the nodes in the information set, such that there exists $y^{t} \rightsquigarrow y$ and $\mu^{t} \rightarrow \mu$ (as $t$ goes to infinity) where the distribution function $\mu^{t}$ is derived from $y^{t}$ using Bayes' rule.

We define each player's perfect and sequential rationality when faced with strategic uncertainty in a game situation as follows.

Definition 1. Consider an extensive form $\Gamma$ with perfect recall. Let $u_{i} \in U_{i}$ and $Y_{-i} \subseteq \mathbb{Y}_{-i}$.

(i) [Perfect Rationality under Uncertainty] Player $i$ 's strategy $y_{i} \in \mathbb{Y}_{i}$ is a perfect best response with respect to $\left(Y_{-i}, u_{i}\right)$ if there exists $y^{t} \rightsquigarrow\left(y_{i}, y_{-i}\right)$ with $y_{-i} \in Y_{-i}$ such that for all $t \geq 0, y_{h} \in \arg \max _{y_{h}^{\prime} \in \mathbb{Y}_{h}} v_{i}\left(\left(y_{h}^{\prime}, y_{-h}^{t}\right), u_{i}\right)$ for all $h \in H_{i}$.

(ii) [Sequential Rationality under Uncertainty] Player $i$ 's strategy $y_{i} \in \mathbb{Y}_{i}$ is a sequential best response with respect to $\left(Y_{-i}, u_{i}\right)$ if there exists a consistent assessment $(y, \mu)$ with $y_{-i} \in Y_{-i}$ such that for all $h \in H_{i}, y _ { i } \in \operatorname { a r g } \operatorname { m a x } _ { y _ { i } ^ { \prime } \in \mathbb { Y } _ { i } } v _ { i } ( y _ { i } ^ { \prime } , ( y , \mu ) , u _ { i } | h ) \longdiv { 1 0 }$

That is, a strategy $y_{i}$ of player $i$ is perfectly rational with respect to $\left(Y_{-i}, u_{i}\right)$ if there exists a trembling sequence $\left\{y^{t}\right\}_{t=0}^{\infty}$ in $\operatorname{int}(\mathbb{Y})$ such that the limit-point profile $y_{-i}=\lim _{t \rightarrow \infty} y_{-i}^{t}$ resides in the uncertain domain $Y_{-i}$, and the limit-point strategy $y_{i}=\lim _{t \rightarrow \infty} y_{i}^{t}$ is a "local" best response under the payoff function $u_{i}$ along the trembling sequence $\left\{y^{t}\right\}_{t=0}^{\infty}$. Similarly, a strategy $y_{i}$ is sequentially rational with respect to $\left(Y_{-i}, u_{i}\right)$ if there exists a consistent assessment $(y, \mu)$ with the constraint $y_{-i} \in Y_{-i}$ such that the strategy $y_{i}$ is a sequential best response under the payoff function $u_{i}$ and consistent assessment $(y, \mu)$. In the certainty case $Y_{-i}=\left\{y_{-i}\right\}$ in the equilibrium paradigm, Definition 1(i) gives rise to perfect rationality in the sense of Selten (1975) and Definition 1(ii) gives rise to sequential rationality (with sequential consistency) in the sense of Kreps and Wilson (1982).

\subsection{MACA: a unifying solution concept}

Consider an extensive form $\Gamma$. Following Dekel et al. (2002), we use the notion of "extensive-form convex hull" to model the subjective uncertainty of a player on the behavioral strategies of an opponent. A behavioral strategy $y_{i}$ of player $i$ is in the extensive-form convex hull of $Y_{i} \subseteq \mathbb{Y}_{i}$, denoted by $\operatorname{co}^{e}\left(Y_{i}\right)$, if

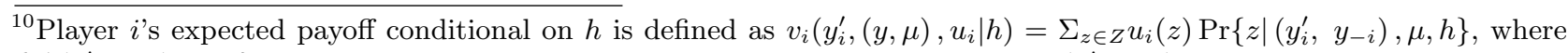
$\operatorname{Pr}\left\{z \mid\left(y_{i}^{\prime}, y_{-i}\right), \mu, h\right\}$ is the probability that $z$ is reached conditionally on $h$ under $\left(y_{i}^{\prime}, y_{-i}\right)$ and $\mu$.
} 
there is a finite set $\left\{y_{i}^{1}, \ldots, y_{i}^{M}\right\} \subseteq Y_{i}$, with trembling sequences $\left(y_{i}^{m, t}\right)_{m=1}^{M} \rightsquigarrow\left(y_{i}^{m}\right)_{m=1}^{M}$ and a sequence $\left(\alpha^{m, t}\right)_{m=1}^{M} \rightarrow \alpha$ of distributions on $\{1, \ldots, M\}$, such that $y_{i}^{t}$ generated by the convex combination $\sum_{m=1}^{M} \alpha^{m, t} y_{i}^{m, t}$ (in terms of realization outcomes) converges to $y_{i}$. A behavioral strategy profile in $\Pi_{j \neq i} c o^{e}\left(Y_{j}\right)$ can be interpreted as an elaborate belief of player $i$ about the opponents' behavior in face of an uncertain domain $\Pi_{j \neq i} Y_{j}$ in the extensive form $\Gamma$ (see Dekel et al. (2002) and Greenberg et al. (2009) for more discussions).

In the context of extensive-form games, Greenberg et al. (2009) offered a unifying solution concept of "mutually acceptable course of action" (MACA) for situations where rational individuals with different beliefs agree to a shared course of action. For the purpose of this paper, we introduce perfect and sequential versions of MACA as follows.

Definition 2 (Greenberg et al. (2009)). In the extensive-form game $\Gamma(u)$ with perfect recall, a course of action $\sigma=\left(\sigma_{h}\right)_{h \in H}$, where $\sigma_{h} \in \mathbb{Y}_{h} \cup\{\emptyset\}$, is a perfect-MACA/sequential-MACA if there exists a set $Y=\prod_{i \in N} Y_{i} \subseteq \mathbb{Y}$ that perfectly/sequentially supports $\sigma$, i.e.,

(i) [agreeing on $\sigma$ ] if $\sigma_{h} \neq \emptyset$, then $y_{h}=\sigma_{h}$ for all $y \in Y$;

(ii) [perfect/sequential rationality] for each $i \in N$, every $y_{i} \in Y_{i}$ is a perfect/sequential best response with respect to $\left(\Pi_{j \neq i} c O^{e}\left(Y_{j}\right), u_{i}\right)$.

Let $\mathrm{T}_{\sigma}^{\text {sequential }}(u) \equiv \cup_{Y}$ sequentially supports $\sigma$ in $\Gamma(u) Y$ denote the union of sequentially $\sigma$-supporting sets in $\Gamma(u)$. Let $\top_{\sigma}^{\text {perfect }}(u) \equiv \cup_{Y}$ perfectly supports $\sigma$ in $\Gamma(u) Y$ denote the union of perfectly $\sigma$-supporting sets in $\Gamma(u)$.

Unlike a behavioral strategy profile, a course of action $\sigma$ does not need to determine a contingent specification of mixed actions at every information set -i.e., it allows to define $\sigma_{h}=\emptyset$ for some information set $h$. Greenberg et al. (2009) demonstrated that, by varying the degree of completeness of the underlying course of action (i.e., the extent of non-null specifications $\sigma_{h} \neq \emptyset$ ), the notion of MACA can be related to commonly used game-theoretic solution concepts, such as equilibrium, self-confirming equilibrium, and rationalizability. More specifically,

- A course of action $\sigma$ is a "complete" perfect-MACA which specifies actions at all information set, i.e. $\sigma_{h} \neq \emptyset \forall h \in H$, if and only if $\sigma$ is a "weakly" perfect equilibrium (in which different players can adopt different sequences of trembles); similarly, $\sigma$ is a "complete" sequential-MACA if and only if $\sigma$ is a "weakly" sequential equilibrium.

- If a course of action $\sigma$ is a "null" perfect-MACA that does not pin down an action at any of the information set, i.e. $\sigma_{h}=\emptyset \forall h \in H$, then the null perfect-MACA $\sigma$ yields a perfect version of rationalizablility, which is related to Herings and Vannetelbosch's (1999) definition 

MACA yields the notion of sequential rationalizability in Dekel et al. (1999, 2002).

- A course of action $\sigma$ is a "path" sequential-MACA, which satisfies $\sigma_{h} \neq \emptyset$ whenever $h$ is reached with positive probability under $\sigma$, if and only if $\sigma$ is a path resulting from sequentially rationalizable self-confirming equilibrium in Dekel et al. (1999, 2002); a "path" perfect-MACA $\sigma$ gives rise to a perfect version of rationalizable self-confirming equilibrium.

\section{Generic equivalence}

Let $\Sigma^{\text {sequential }}(u)$ and $\Sigma^{\text {perfect }}(u)$ denote the sets of sequential-MACAs and perfect-MACAs in $\Gamma(u)$, respectively. Define the sequential-MACA-cluster correspondence $\Upsilon^{\text {sequential }}: U \rightrightarrows \Pi_{\mathcal{H} \in 2^{H} \backslash\{\emptyset\}}\left[\Pi_{h \in \mathcal{H}} \mathbb{Y}_{h}\right]$ and perfect-MACA-cluster correspondence $\Upsilon^{\text {perfect }}: U \rightrightarrows \Pi_{\mathcal{H} \in 2^{H} \backslash\{\emptyset\}}\left[\Pi_{h \in \mathcal{H}} \mathbb{Y}_{h}\right]$ (where $2^{H}$ is the power set of $H)$ as follows: For all $u \in U$,

$$
\begin{aligned}
\Upsilon^{\text {sequential }}(u) & \equiv \prod_{\mathcal{H} \in 2^{H} \backslash\{\emptyset\}}\left\{\left(y_{h}\right)_{h \in \mathcal{H}} \in \Pi_{h \in \mathcal{H}} \mathbb{Y}_{h}: \exists \sigma \in \Sigma^{\text {sequential }}(u) \text { s.t. } \sigma_{h}=\left\{\begin{array}{ll}
y_{h}, & \text { if } h \in \mathcal{H} \\
\emptyset, & \text { otherwise }
\end{array}\right\} ;\right. \\
\Upsilon^{\text {perfect }}(u) & \equiv \prod_{\mathcal{H} \in 2^{H} \backslash\{\emptyset\}}\left\{\left(y_{h}\right)_{h \in \mathcal{H}} \in \Pi_{h \in \mathcal{H}} \mathbb{Y}_{h}: \exists \sigma \in \Sigma^{\text {perfect }}(u) \text { s.t. } \sigma_{h}=\left\{\begin{array}{ll}
y_{h}, & \text { if } h \in \mathcal{H} \\
\emptyset, & \text { otherwise }
\end{array}\right\} .\right.
\end{aligned}
$$

This notation $\Upsilon^{\text {sequential }}(u)$ is naturally related to the Cartesian product of clusters of (nonnull) sequential-MACAs in $\Gamma(u)$, where a cluster is a bunch of the sequential MACAs that can each determine a contingent specification of mixed actions only for a particular collection of information sets in $\Gamma$; the notation $\Upsilon^{\text {perfect }}(u)$ is related to a Cartesian product of clusters of (nonnull) perfect-MACAs in $\Gamma(u)$ in the same manner. For instance, the set of all the complete sequential-MACAs in $\Gamma(u)$ is simply a cluster of sequential equilibria in $\Gamma(u)$. We are now in a position to present the central result of this paper.

Theorem 1. Consider an extensive form $\Gamma$ with perfect recall. There is a closed, lower-dimensional semi-algebraic subset $U^{0} \subset U$ such that for all $u \in U \backslash U^{0}$, in game $\Gamma(u)$, (i) the set of sequentialMACAs coincides with the set of perfect-MACAs (i.e., $\Sigma^{\operatorname{sequential}}(u)=\Sigma^{\text {perfect }}(u)$ ) and (ii) for an arbitrary given course of action $\sigma$ in $\Gamma$, the union of sequentially $\sigma$-supporting sets coincides with the union of perfectly $\sigma$-supporting sets (i.e., $\top_{\sigma}^{\text {sequential }}(u)=\top_{\sigma}^{\text {perfect }}(u)$ ).

Moreover, $\Upsilon^{\text {sequential }}(u)=\Upsilon^{\text {perfect }}(u)$ for all the payoffs $u \in U$ at which the sequential-MACAcluster correspondence $\Upsilon^{\text {sequential }}(\cdot)$ is lower hemi-continuous and the perfect-MACA-cluster correspondence $\Upsilon^{\text {perfect }}(\cdot)$ is upper hemi-continuous. In addition, for an arbitrary given course of action $\sigma$ in $\Gamma, \top_{\sigma}^{\text {sequential }}(u)=\top_{\sigma}^{\text {perfect }}(u)$ for all the payoffs $u \in U$ at which $\top_{\sigma}^{\text {sequential }}(\cdot)$ is lower hemi-continuous and $\top_{\sigma}^{\text {perfect }}(\cdot)$ is upper hemi-continuous. 

of strategy $y_{i}$ is optimal with respect to payoff function $u_{i} \in U_{i}$ and (cautious) belief $x \in \operatorname{int}(\mathbb{Y}$ ). In the spirit of Kreps and Wilson (1982), we provide a useful characterization of perfect rationality and sequential rationality respectively by the closure and vertical closure of $\mathcal{R}_{i}$. More specifically, player $i$ 's strategy $y_{i}$ is a sequential (resp. perfect) best response with respect to $\left(Y_{-i}, u_{i}\right)$ if, and only if, $y_{i}$ can be embedded in the closure (resp. vertical closure) of $\mathcal{R}_{i}$ with the restriction that the limit-point belief is consistent with the opponents' choices in $c o^{e}\left(Y_{-i}\right)$ and his own choice $y_{i}$ (Lemma 1 in Appendix B). ${ }^{11}$ By the Tarski-Seidenberg Theorem, $\mathcal{R}_{i}$ is a semi-algebraic set. We then adopt the methods in BZ94 to show that the closure and vertical closure of $\mathcal{R}_{i}$ are generically equivalent (Lemma 2 in Appendix B), and establish an elementary generic equivalence between perfect rationality and sequential rationality for an individual player in an extensive form (Lemma 3 in Appendix B). By using the analytical framework of MACA, we can obtain a very general generic equivalence between perfectly and sequentially rational behavior, under the elaborate belief structure that each player holds an "independent" limit-point belief about each opponent player's behavior residing in the extensive-form convex hull of an uncertain domain of behavioral strategies.

In contrast to the BZ94 approach that relies crucially on the semi-algebraic property of the equilibrium solution concepts, our approach does not depend directly on the semi-algebraic property of $a d$ hoc solution concepts. Our analysis is solely based on the semi-algebraic property of the elementary set $\mathcal{R}_{i}$; thus it is feasible and applicable to a variety of game-theoretic solution concepts, as long as the trembling sequence in the analysis of perfectly and sequentially rational behavior is confirmed to be concordant with the belief structure adopted in an extensive-form game. If we work with a belief structure in which every player holds a consistently aligned cautious belief about the players' behavior in the equilibrium paradigm, our approach delivers the BZ94 generic equivalence result for the notions of sequential and perfect equilibria (see Appendix A for their definitions).

Corollary 1 (BZ94, Theorem 4). Consider an extensive form $\Gamma$ with perfect recall. There is a closed, lower-dimensional semi-algebraic subset $U^{0} \subset U$ such that for all $u \in U \backslash U^{0}$, any sequential equilibrium strategy profile is a perfect equilibrium in game $\Gamma(u)$. Moreover, the sets of perfect and sequential equilibrium strategy profiles coincide for all the payoffs $u \in U$ at which the sequential equilibrium correspondence is lower hemi-continuous and the perfect equilibrium correspondence is upper hemicontinuous.

\footnotetext{
${ }^{11}$ To relate to Selten's (1975) perfectness, Kreps and Wilson (1982, Proposition 6) provided a useful characterization of sequential equilibrium in terms of "payoff perturbations"; they relaxed Selten's criterion by allowing some (vanishingly) small uncertainty on the part of players' payoffs (cf. also Halpern (2009)).
} 
By varying the degree of completeness of the underlying course of action, we obtain an immediate corollary of Theorem 1 as follows (cf. Appendix A for definitions).

Corollary 2. Consider an extensive form $\Gamma$ with perfect recall. There is a closed, lower-dimensional semi-algebraic subset $U^{0} \subset U$ such that for all $u \in U \backslash U^{0}$, in game $\Gamma(u)$,

(i) the set of weakly sequential equilibria coincides with the set of weakly perfect equilibria;

(ii) the set of sequentially rationalizable strategy profiles (Dekel et al. (1999, 2002)) coincides with the set of perfectly rationalizable strategy profiles (Greenberg et al. (2009));

(iii) the set of paths resulting from sequentially rationalizable self-confirming equilibria (Dekel et al. (1999, 2002)) coincides with the set of path perfect-MACAs (Greenberg et al. (2009)).

Moreover, for all $u \in U \backslash U^{0}$, the largest (w.r.t. set inclusion) sequentially $\sigma$-supporting set in $\Gamma(u)$ coincides with the largest perfectly $\sigma$-supporting set in $\Gamma(u)$.

Remark. Fudenberg and Levine (1993) introduced the notion of self-confirming equilibrium that is a generalization of Nash equilibrium for extensive-form games, in which players correctly predict the actions their opponents take along the equilibrium path, but may have misconceptions about what their opponents would do at information sets that are never reached when the equilibrium is played (see also Fudenberg and Levine (2006; 2009), Esponda (2013), Battigalli et al. (2015), and Fudenberg and Kamada (2015)) ${ }^{12}$ Dekel et al. (1999, 2002) developed a refinement of sequentially rationalizable self-confirming equilibrium (SRSCE), which is related to assuming sequential rationality and almost common certainty of payoffs in an epistemic model with independent beliefs (cf. Asheim and Perea (2005) and Luo and Wang (2017)). Dekel et al. (1999, Footnote 4) claimed the "generic" equivalence between SRSCE and the path perfect-MACA in Greenberg et al. (2009), but they offered no formal analysis of the claim. Corollary 2(iii) shows this claim is true.

We end this section by providing an application of our approach in extensive forms with simultaneous moves. Corollary 3 below asserts that, in normal form games, iterated elimination of weakly dominated strategies (IEWDS) is generically an order-independent procedure which is equivalent to iterated elimination of strictly dominated strategies (IESDS) ${ }^{13}$

\footnotetext{
${ }^{12}$ The notion is also related to Rubinstein and Wolinsky's (1994) notion of a "rationalizable conjectural equilibrium" ( $\mathrm{RCE})$.

${ }^{13}$ It is worth noting that in the generic set $U \backslash U^{0}$ (Corollary 3), there are games with strictly/weakly dominated strategies, so that the statement of Corollary 3 is not vacuously true. The major reason is that if a strategy is strictly dominated at a payoff vector $u \in U$, then this is also true in a neighborhood of $u$; hence, strictly dominated strategies exist in a full-dimensional payoff subset (excluding the lower-dimensional set $U^{0}$ ). We thank an anonymous referee for drawing our attention to this point.
} 
Corollary 3. Consider a (finite) normal form $\Gamma$. There exists a closed, lower-dimensional semialgebraic subset $U^{0} \subset U$ such that for all $u \in U \backslash U^{0}$, every IEWDS procedure is an IESDS procedure; hence, IEWDS is generically an order-independent procedure.

\section{Concluding remarks}

BZ94 strengthened Kreps and Wilson's (1982) result and showed that, for almost all assignments of payoffs to the terminal nodes, the sets of sequential and perfect equilibria are identical. In this paper, we have extended BZ94's result to more general settings of strategic interactions. We have formulated and proved a general and fundamental generic equivalence between sequential and perfect rational behavior in extensive-form games. By using Greenberg et al.'s (2009) concept of MACA, we have established a unified generic equivalence between the notions of sequential MACA and perfect MACA (Theorem 1). We have demonstrated that we can obtain a variety of generic equivalence results for various kinds of solution concepts such as equilibrium, rationalizability, and self-confirming equilibrium, as corollaries of our general generic equivalence theorem. The study of this paper helps deepen our understanding of the fine structural relationship between perfectly and sequentially rational strategic behavior under different behavioral assumptions.

In this paper, we have followed Dekel et al. $(1999,2002)$ and Greenberg et al. (2009) to adopt a simple and convenient way of defining perfect/sequential rationality by using sequences of trembles and present a unified framework for the study of the generic relationship between perfectly and sequentially rational strategic behavior in a broad sense. Alternatively, one may follow BZ94's approach to analyze perfectly and sequentially rational strategic behavior by using "perturbed games." However, there is no formal formulation of perfect/sequential rationalizability for extensive-form games, in terms of perturbed games, in the literature, although Bernheim (1984, pp.1021-1022) outlined such a notion of perfect rationalizability in normal form games. Herings and Vannetelbosch (1999, Example G7) showed that, unlike the notion of perfect equilibrium, there are different definitions of perfect rationalizability by using "trembling conjectures" or "perturbed games" (cf. also Börgers (1994)). In particular, the alternative definition of perfect/sequential rationalizability by using "perturbed games" may suffer Fudenberg and Tirole's (1991) criticism: It implicitly requires that all players have the same theory to form common "trembling conjectures," as illustrated by the following example.

It is easy to see that the strategy profile $y=\left(E_{1}, C_{2}, R_{3}\right)$, marked by bold lines in Figure 2 , is sequentially/perfectly rationalizable. But, the profile $y$ is not sequentially/perfectly rationalizable in terms of "perturbed games". To see this, note that (i) in any perturbed game, because $E_{1}$ strictly dominates $L_{1}$ and $R_{1}$, there is a unique rationalizable strategy for player 1 - i.e., playing $L_{1}$ and $R_{1}$ with the minimum probabilities specified in the perturbed game, and (ii) player 2 and 3 must hold common "trembling conjectures" in commonly known "perturbed games". But, $C_{2}$ is sequentially rational only if $p \leq 1 / 3 ; R_{3}$ is sequentially rational only if $q \geq 2 / 3$. Subsequently, the profile $\left(C_{2}, R_{3}\right)$ cannot be sequentially/perfectly rationalizable in terms of "perturbed games". This argument is valid for a neigh- 
borhood of the payoffs to the terminal nodes 14 Since this kind of implicit requirement of common "trembling conjectures" appears to be less convincing and arguable especially in a non-equilibrium setting, we do not use this alternative way of formulating perfectly/sequentially rational strategic behavior in the perturbed games in this paper.

As we have emphasized, unlike BZ94's approach, our analysis in this paper does not rely directly on semi-algebraic properties of specific solutions concepts (e.g., the semi-algebraic structure of perfect and sequential equilibrium correspondences in BZ94). Instead, our approach is based simply on the primitive set $\mathcal{R}_{i}$ of "perfectly-rational" states, so that it is feasible and applicable to various solution concepts under different behavioral assumptions. We believe that our approach to the generic equivalence relation provides a useful and complementary way for the study of the relationship between perfectly and sequentially rational strategic behavior in a wide range of environments.

Through the lens of Greenberg et al.'s (2009) concept of MACA, Theorem 1 gives rise to a generic equivalence between Dekel et al.'s $(1999,2002)$ sequential rationalizability and Greenberg et al.'s (2009) perfect rationalizability with an elaborate belief structure, namely the extensive-form convex hull (Corollary 2 (ii)). We would like to point out that our analysis is valid for a special kind of pointbelief structure in which each player holds a degenerate point-mass belief about the opponent players' uncertain behavior (cf. Bernheim (1984) for the notion of point-rationalizability); that is, the sequential and perfect versions of point-rationalizability are generically equivalent. There are other definitions of rationalizability in the literature, e.g., Pearce's (1984) extensive form rationalizability (EFR). Battigalli (1997), Perea (2018), Brandenburger and Friedenberg (2003), and Shimoji (2004) studied related issues on the equivalence of weak dominance and sequential best response; they showed that EFR and IEWDS are "outcome" equivalent in generic complete-information games. The extension of our paper to the solution concept of EFR is certainly an important and interesting subject for further research, but beyond the scope of this paper. In this paper, we restrict our attention to player $i$ 's independent beliefs in $\Pi_{j \neq i} c o^{e}\left(Y_{j}\right)$ about the opponents' behavioral strategies. How to extend this paper to the situations

\footnotetext{
${ }^{14}$ This example shows that the notions of perfect Bayesian equilibrium and sequential equilibrium are generically distinct, because $\left(E_{1}, C_{2}, R_{3}\right)$ is a perfect Bayesian equilibrium but not a sequential equilibrium.
} 
by allowing correlated beliefs about the opponents' behavioral strategies is also an intriguing subject for future research. 


\section{Appendix A: Definitions}

Consider an extensive-form game $\Gamma(u)$ with perfect recall.

Definition A1. (i) [Selten (1975); Osborne and Rubinstein (1994, Definition 251.1)] A strategy profile $y \in \mathbb{Y}$ is a perfectly equilibrium if there exists a trembling sequence $y^{t} \rightsquigarrow y$ such that, for all $t \geq 0, y_{h} \in \arg \max _{y_{h}^{\prime} \in \mathbb{Y}_{h}} v_{i}\left(\left(y_{h}^{\prime}, y_{-h}^{t}\right), u_{i}\right)$ for all $i \in N$ and $h \in H_{i}$.

(ii) [Kreps and Wilson (1982)] A strategy profile $y \in \mathbb{Y}$ is a sequential equilibrium if there exists a consistent assessment $(y, \mu)$ such that $y_{i} \in \arg \max _{y_{i}^{\prime} \in \mathbb{Y}_{i}} v_{i}\left(y_{i}^{\prime},(y, \mu), u_{i} \mid h\right)$ for all $i \in N$ and $h \in H_{i}$.

(iii) A strategy profile $y \in \mathbb{Y}$ is a "weakly" perfect equilibrium if for each player $i \in N$, there exists a trembling sequence $y^{t} \rightsquigarrow y$ such that, for all $t \geq 0, y_{h} \in \arg \max _{y_{h}^{\prime} \in \mathbb{Y}_{h}} v_{i}\left(\left(y_{h}^{\prime}, y_{-h}^{t}\right), u_{i}\right)$ for all $h \in H_{i}$.

(iv) A strategy profile $y \in \mathbb{Y}$ is a "weakly" sequential equilibrium if for each player $i \in N$, there exists a consistent assessment $(y, \mu)$ such that $y_{i} \in \arg \max _{y_{i}^{\prime} \in \mathbb{Y}_{i}} v_{i}\left(y_{i}^{\prime},(y, \mu), u_{i} \mid h\right)$ for all information set $h \in H_{i}$.

In the notions of perfect equilibrium and sequential equilibrium, all the players are required to have a (common) trembling sequence. In contrast, in the notions of "weakly" perfect equilibrium and "weakly" sequential equilibrium, different players are allowed to adopt distinct trembling sequences 15

Definition A2 (Greenberg et al. (2009) and Dekel et al. (1999, 2002)). A product set $Y=\Pi_{i \in N} Y_{i} \subseteq \mathbb{Y}$ is a perfectly (or sequentially) rationalizable set if for each player $i \in N$, every $y_{i} \in Y_{i}$ is perfect (or sequential) best response with respect to $\left(\Pi_{j \neq i} c o^{e}\left(Y_{j}\right), u_{i}\right)$. An element in a perfectly (or sequentially) rationalizable set is said to be a perfectly (or sequentially) rationalizable strategy profile.

That is, $Y$ is a perfectly rationalizable set if, for each player $i$ and $y_{i} \in Y_{i}$, we can find a strategy profile $y_{-i} \in \Pi_{j \neq i} c O^{e}\left(Y_{j}\right)$ and a trembling sequence which converges to $\left(y_{i}, y_{-i}\right)$ such that $y_{i}$ is a best response along the trembling sequence. In simultaneous-move games, the perfectly rationalizable set is related to Herings and Vannetelbosch's (1999) definition of "weakly perfect rationalizability." Similarly, $Y$ is a sequentially rationalizable set if for each player $i$ and $y_{i} \in Y_{i}$, we can find a consistent assessment $\left(\left(y_{i}, y_{-i}\right), \mu\right)$ where $y_{-i} \in \Pi_{j \neq i} c O^{e}\left(Y_{j}\right)$ such that $y_{i}$ is a sequential best response. The sequentially rationalizable set is associated with Dekel et al.'s (1999, 2002) notion of sequential rationalizablility.

Definition A3 (Dekel et al. (1999, 2002)). A strategy profile $\widehat{y} \in \mathbb{Y}$ is a sequentially rationalizable self-confirming equilibrium (SRSCE) if there exists $Y=\Pi_{i \in N} Y_{i} \subseteq \mathbb{Y}$, such that for each $i \in N$ and $y_{i} \in Y_{i}$, we can find a consistent assessment $(y, \mu)$, with the restrictions that (i) $y_{-i} \in \Pi_{j \neq i} c o^{e}\left(Y_{j}\right)$, (ii)

\footnotetext{
${ }^{15}$ By allowing for distinct trembling sequences for different players, Aryal and Stauber (2014) introduced the notions of trembling-hand perfect equilibrium and robust sequential equilibrium in extensive games with ambiguity averse players.
} 
$y$ and $\widehat{y}$ yield a same distribution over terminal nodes, and (iii) $y_{i}$ is a sequential best response under $(y, \mu)$.

\section{Appendix B: Proofs}

For each player $i \in N$ and $Y_{-i} \subseteq \mathbb{Y}_{-i}$, let

$$
\begin{aligned}
P B_{i}\left(Y_{-i}, u_{i}\right) & \equiv\left\{y_{i} \in \mathbb{Y}_{i}: y_{i} \text { is a perfect best response with respect to }\left(Y_{-i}, u_{i}\right)\right\} \\
S B_{i}\left(Y_{-i}, u_{i}\right) & \equiv\left\{y_{i} \in \mathbb{Y}_{i}: y_{i} \text { is a sequential best response with respect to }\left(Y_{-i}, u_{i}\right)\right\} .
\end{aligned}
$$

Let $\operatorname{cl}\left(\mathcal{R}_{i}\right)$ and $v c l_{U_{i}}\left(\mathcal{R}_{i}\right)$ denote the closure of the perfectly-rational state set $\mathcal{R}_{i}$ and vertical closure of the perfectly-rational state set $\mathcal{R}_{i}$ (on $U_{i}$ ), respectively; that is,

$$
\begin{aligned}
c l\left(\mathcal{R}_{i}\right) & \equiv\left\{\left(u_{i}, x, y_{i}\right) \in U_{i} \times \mathbb{Y} \times \mathbb{Y}_{i}: \exists\left\{\left(u_{i}^{t}, x^{t}, y_{i}^{t}\right)\right\}_{t=1}^{\infty} \in \mathcal{R}_{i} \text { s.t. }\left(u_{i}^{t}, x^{t}, y_{i}^{t}\right) \rightarrow\left(u_{i}, x, y_{i}\right)\right\} \\
v c l_{U_{i}}\left(\mathcal{R}_{i}\right) & \equiv\left\{\left(u_{i}, x, y_{i}\right) \in U_{i} \times \mathbb{Y} \times \mathbb{Y}_{i}: \exists\left\{\left(u_{i}, x^{t}, y_{i}^{t}\right)\right\}_{t=1}^{\infty} \in \mathcal{R}_{i} \text { s.t. }\left(u_{i}, x^{t}, y_{i}^{t}\right) \rightarrow\left(u_{i}, x, y_{i}\right)\right\} .
\end{aligned}
$$

Let

$$
Q\left(Y_{-i}\right) \equiv\left\{\left(u_{i}, x, y_{i}\right) \in U_{i} \times \mathbb{Y} \times \mathbb{Y}_{i}: x_{i}=y_{i} \text { and } x_{-i} \in Y_{-i}\right\}
$$

denote the set of "consistent-belief" states at which player $i$ 's belief about the opponent players' behavior lies in the given set $Y_{-i}$ and the belief about his own behavior is consistent with his strategy.

To prove Theorem 1, we need the following four lemmas.

Lemma 1. In an extensive form $\Gamma$ with perfect recall, for each player $i \in N$ and $Y_{-i} \subseteq \mathbb{Y}_{-i}$ (a) $y_{i} \in$ $P B_{i}\left(Y_{-i}, u_{i}\right) \Leftrightarrow \exists\left(u_{i}, x, y_{i}\right) \in \operatorname{vcl}_{U_{i}}\left(\mathcal{R}_{i}\right) \cap Q\left(Y_{-i}\right) ;(b) y_{i} \in S B_{i}\left(Y_{-i}, u_{i}\right) \Leftrightarrow \exists\left(u_{i}, x, y_{i}\right) \in \operatorname{cl}\left(\mathcal{R}_{i}\right) \cap Q\left(Y_{-i}\right)$.

Proof: (a) Suppose $y_{i} \in P B_{i}\left(Y_{-i}, u_{i}\right)$. Then, there exist $y_{i}^{t} \rightsquigarrow y_{i}$ and $x_{-i}^{t} \rightsquigarrow x_{-i} \in Y_{-i}$ such that $\left(u_{i},\left(y_{i}^{t}, x_{-i}^{t}\right), y_{i}\right) \in \mathcal{R}_{i}$ for all $t$. Since $\left(u_{i},\left(y_{i}^{t}, x_{-i}^{t}\right), y_{i}\right) \rightarrow\left(u_{i},\left(y_{i}, x_{-i}\right), y_{i}\right),\left(u_{i}, x, y_{i}\right) \in v c l_{U_{i}}\left(\mathcal{R}_{i}\right) \cap$ $Q\left(Y_{-i}\right)$. Conversely, suppose $\left(u_{i}, x, y_{i}\right) \in \operatorname{vcl}_{U_{i}}\left(\mathcal{R}_{i}\right), x_{-i} \in Y_{-i}$ and $x_{i}=y_{i}$. Then there exists a sequence $\left(u_{i}, x^{t}, y_{i}^{t}\right) \in \mathcal{R}_{i}$ converging to $\left(u_{i}, x, y_{i}\right)$. Since $\Gamma$ is finite and $y_{i}^{t} \rightarrow y_{i}$, there is a sufficiently large $T$ such that, for all $t \geq T$ and $h \in H_{i}, a_{h} \in \operatorname{support}\left(y_{h}\right) \operatorname{implies} a_{h} \in \operatorname{support}\left(y_{h}^{t}\right)$ and $a_{h} \in$ $\arg \max _{a_{h}^{\prime} \in A_{h}} v_{i}\left(\left(a_{h}^{\prime}, x_{-h}^{t}\right), u_{i}\right)$. Therefore, $y_{h} \in \arg \max _{y_{h}^{\prime} \in \mathbb{Y}_{h}} v_{i}\left(\left(y_{h}^{\prime}, x_{-h}^{t}\right), u_{i}\right)$ for all $t \geq T$ and all $h \in H_{i}$. That is, $y_{i} \in P B_{i}\left(Y_{-i}, u_{i}\right)$.

(b) It suffices to show $y_{i} \in S B_{i}\left(Y_{-i}, u_{i}\right)$ iff there exist $u_{i}^{t} \rightarrow u_{i}$ and $\left(y_{i}^{t}, x_{-i}^{t}\right) \rightsquigarrow\left(y_{i}, x_{-i}\right)$ such that $x_{-i} \in Y_{-i}, y_{h} \in \arg \max _{y_{h}^{\prime} \in \mathbb{Y}_{h}} v_{i}\left(\left(y_{h}^{\prime}, x_{-h}^{t}\right), u_{i}^{t}\right)$ for all $t$ and all $h \in H_{i}$.

$" \Leftarrow "$ : Let $x^{t}=\left(y_{i}^{t}, x_{-i}^{t}\right) \rightsquigarrow\left(y_{i}, x_{-i}\right)=x$ such that $x_{-i} \in Y_{-i}$. Without loss of generality, assume $\left(x^{t}, \mu_{i}^{t}\right) \rightarrow\left(x, \mu_{i}\right)$, where $\mu_{i}^{t}$ is derived from $x^{t}$ using Bayes' rule. Suppose that there exist $u_{i}^{t} \rightarrow u_{i}$ such that $y_{h} \in \arg \max _{y_{h}^{\prime} \in \mathbb{Y}_{h}} v_{i}\left(\left(y_{h}^{\prime}, x_{-h}^{t}\right), u_{i}^{t}\right)$ for all $t$ and all $h \in H_{i}$. Then, for all 
$h \in H_{i}$ and all $t, v_{i}\left(\left(y_{h}, x_{-h}^{t}\right), u_{i}^{t} \mid h\right) \geq v_{i}\left(\left(y_{h}^{\prime}, x_{-h}^{t}\right), u_{i}^{t} \mid h\right) \forall y_{h}^{\prime} \in \mathbb{Y}_{h} \cdot{ }^{16}$ Since $v_{i}\left(\left(y_{h}, \cdot\right), \cdot \mid h\right)$ is continuous, $v_{i}\left(\left(y_{h}, x_{-h}\right), u_{i} \mid h\right) \geq v_{i}\left(\left(y_{h}^{\prime}, x_{-h}\right), u_{i} \mid h\right)$. Since $\Gamma$ is perfect recall, every sequential optimal strategy satisfies the one deviation property (see, Perea 2002), for all $h \in H_{i}, v_{i}\left(\left(y_{i}, x_{-i}\right), u_{i} \mid h\right) \geq$ $v_{i}\left(\left(y_{i}^{\prime}, x_{-i}\right), u_{i} \mid h\right) \forall y_{i}^{\prime} \in \mathbb{Y}_{i}$. That is, $y_{i} \in S B_{i}\left(Y_{-i}, u_{i}\right)$.

$" \Rightarrow "$ : Let $y_{i} \in S B_{i}\left(Y_{-i}, u_{i}\right)$. Then, there is $\left(\left(y_{i}^{t}, x_{-i}^{t}\right), \mu_{i}^{t}\right) \rightarrow\left(\left(y_{i}, x_{-i}\right), \mu_{i}\right)$ such that $x_{-i} \in$ $Y_{-i}$ and $y_{i}$ is sequentially optimal against the assessment $\left(\left(y_{i}, x_{-i}\right), \mu_{i}\right)$. Denote $\left(y_{i}, x_{-i}\right)=x$ and $\left(y_{i}^{t}, x_{-i}^{t}\right)=x^{t}$. Clearly, $x^{t} \rightsquigarrow x$. We proceed to construct a payoff sequence $u_{i}^{t} \rightarrow u_{i}$ such that $y_{h} \in \arg \max _{y_{h}^{\prime} \in \mathbb{Y}_{h}} v_{i}\left(\left(y_{h}^{\prime}, x_{-h}^{t}\right), u_{i}^{t}\right)$ for all $t$ and all $h \in H_{i}$.

Since $\Gamma$ is finite and perfect recall holds, we can define a (finite) partition $\left\{H_{i}^{l}\right\}_{l=1}^{L}$ of the set $H_{i}$ as follows: $H_{i}^{0}=\varnothing, H_{i}^{l} \equiv\left\{h \in H_{i} \backslash \cup_{\ell<l} H_{i}^{\ell}:\right.$ no $h^{\prime} \in\left[H_{i} \backslash \cup_{\ell<l} H_{i}^{\ell}\right] \backslash h$ is reached by $\left.h\right\}$ for all $l \geq 1$. Therefore, for all $t$ and $l=1, \cdots, L$, we can define $u_{i}^{t, l}$ recursively as follows: Let $u_{i}^{t, 0} \equiv u_{i}$,

$$
u_{i}^{t, l}(z) \equiv\left\{\begin{array}{ll}
u_{i}^{t, l-1}(z)+\delta_{a_{h}^{*}}^{t}, & \text { if } z \text { is not precluded by } a_{h}^{*} \in \operatorname{support}\left(y_{h}\right) \text { from } h \\
u_{i}^{t, l-1}(z), & \text { otherwise }
\end{array},\right.
$$

where $h \in H_{i}^{l}, \operatorname{support}\left(y_{h}\right)=\left\{a_{h} \in A_{h}: y_{h}\left(a_{h}\right)>0\right\}$ and

$$
\delta_{a_{h}^{*}}^{t}=\max _{a_{h} \in A_{h}} v_{i}\left(\left(a_{h}, x_{-h}^{t}\right), u_{i}^{t, l-1} \mid h\right)-v_{i}\left(\left(a_{h}^{*}, x_{-h}^{t}\right), u_{i}^{t, l-1} \mid h\right) .
$$

Therefore, for $l=1, \cdots, L, y_{h} \in \arg \max _{y_{h}^{\prime} \in \mathbb{Y}_{h}} v_{i}\left(\left(y_{h}^{\prime}, x_{-h}^{t}\right), u_{i}^{t, l} \mid h\right) \forall h \in H_{i}^{l}$. For all $a_{h}, a_{h}^{\prime} \in A_{h}$, $v_{i}\left(\left(a_{h}, x_{-h}^{t}\right), u_{i}^{t, l+1} \mid h\right)-v_{i}\left(\left(a_{h}^{\prime}, x_{-h}^{t}\right), u_{i}^{t, l+1} \mid h\right)=v_{i}\left(\left(a_{h}, x_{-h}^{t}\right), u_{i}^{t, l} \mid h\right)-v_{i}\left(\left(a_{h}^{\prime}, x_{-h}^{t}\right), u_{i}^{t, l} \mid h\right)$. By induction on $l$, we have $y_{h} \in \arg \max _{y_{h}^{\prime} \in \mathbb{Y}_{h}} v_{i}\left(\left(y_{h}^{\prime}, x_{-h}^{t}\right), u_{i}^{t, l}\right) \forall h \in \cup_{\ell=1}^{l} H_{i}^{\ell}$. Hence, $y_{h} \in$ $\arg \max _{y_{h}^{\prime} \in \mathbb{Y}_{h}} v_{i}\left(\left(y_{h}^{\prime}, x_{-h}^{t}\right), u_{i}^{t, L}\right) \forall h \in H_{i}$, which implies that $\left(u_{i}^{t},\left(y_{i}^{t}, x_{-i}^{t}\right), y_{i}\right) \in \mathcal{R}_{i}$.

It remains to show $u_{i}^{t, L} \rightarrow u_{i}^{L}=u_{i}$. We prove this by induction on $l$. Clearly, $u_{i}^{t, 0} \rightarrow u_{i}$ trivially holds. Suppose $u_{i}^{t, \ell} \rightarrow u_{i}^{\ell}=u_{i}$ for $\ell \leq l-1$. By construction of $u_{i}^{t, l}$, it suffices to show $\delta_{a_{h}^{*}}^{t} \rightarrow 0 \forall h \in H_{i}^{l}$. Let $\hat{a}_{h} \in \arg \max _{a_{h} \in A_{h}} v_{i}\left(\left(a_{h}, x_{-h}^{t}\right), u_{i}^{t, l-1} \mid h\right)$. Because of the continuity of $v_{i}$, for any $\varepsilon>0$ there is a sufficiently large $T$ such that, for all $t>T$,

$$
\begin{aligned}
& v_{i}\left(\left(\hat{a}_{h}, x_{-h}^{t}\right), u_{i}^{t, l-1} \mid h\right)-v_{i}\left(\left(\hat{a}_{h}, x_{-h}\right), u_{i}^{l-1} \mid h\right)<\varepsilon \\
& v_{i}\left(\left(a_{h}^{*}, x_{-h}\right), u_{i}^{l-1} \mid h\right)-v_{i}\left(\left(a_{h}^{*}, x_{-h}^{t}\right), u_{i}^{t, l-1} \mid h\right)<\varepsilon .
\end{aligned}
$$

Since $y_{h} \in \arg \max _{y_{h}^{\prime} \in \mathbb{Y}_{h}} v_{i}\left(\left(y_{h}^{\prime}, x_{-h}\right), u_{i}^{t}\right)$ and, by the induction hypothesis, $u_{i}=u_{i}^{l-1}$,

$$
v_{i}\left(\left(\hat{a}_{h}, x_{-h}\right), u_{i}^{l-1} \mid h\right)-v_{i}\left(\left(a_{h}^{*}, x_{-h}\right), u_{i}^{l-1} \mid h\right) \leq 0 .
$$

\footnotetext{
$\left.\overline{{ }^{16} \text { For any } y_{i}^{\prime} \in \mathbb{Y}_{i} \text {, we define } v_{i}\left(\left(y_{i}^{\prime}, x_{-i}^{t}\right), u_{i}^{t} \mid h\right.}\right) \equiv v_{i}\left(y_{i}^{\prime},\left(x_{-i}^{t}, \mu_{i}^{t}\right), u_{i}^{t} \mid h\right)$.
} 
Lemma 2: Let $W \subseteq \mathbb{R}^{n+m}$ be a semi-algebraic set. (a) cl $(W)$ and $v \operatorname{ll}_{\mathbb{R}^{n}}(W)$ are semi-algebraic. (b) There exists a closed, lower-dimensional semi-algebraic subset $E \subset \mathbb{R}^{n}$ such that for all $x \in \mathbb{R}^{n} \backslash E$, $\left\{y \in \mathbb{R}^{m}:(x, y) \in \operatorname{cl}(W)\right\}=\left\{y \in \mathbb{R}^{m}:(x, y) \in \operatorname{vcl}_{\mathbb{R}^{n}}(W)\right\}$.

Proof: (a) $v c l_{\mathbb{R}^{n}}(W)$ can be rewritten as

$$
\left\{(x, y) \in \mathbb{R}^{n} \times \mathbb{R}^{m}: \forall \varepsilon>0, \exists\left(x, y^{\prime}\right) \in W \text { s.t. }\left\|y-y^{\prime}\right\|<\varepsilon\right\}
$$

where $\|\cdot\|$ is the Euclidean norm. Since $W$ is semi-algebraic, it follows from Tarski-Seidenberg Theorem that $v c \mathbb{R}_{\mathbb{R}^{n}}(W)$ is also semi-algebraic. Similarly, $c l(W)$ is semi-algebraic.

(b) This proof is similar to the proof of Theorem 4 in BZ94. Define $f, g: \mathbb{R}^{n} \times \mathbb{R}^{m} \rightarrow \mathbb{R}$ by

$$
\begin{aligned}
& f(x, y) \equiv \inf _{\left(x^{\prime}, y^{\prime}\right) \in W}\left\|(x, y)-\left(x^{\prime}, y^{\prime}\right)\right\| \\
& g(x, y) \equiv \inf _{\left(x, y^{\prime}\right) \in W}\left\|(x, y)-\left(x, y^{\prime}\right)\right\|
\end{aligned}
$$

where $x$ and $x^{\prime}$ belong to $\mathbb{R}^{n}, y$ and $y^{\prime}$ belong to $\mathbb{R}^{m}$.

Define

$$
E=\left\{x \in \mathbb{R}^{n}: \exists(x, y) \in W, f(x, y)=0 \text { and } g(x, y)>0\right\} .
$$

Since $f$ and $g$ are semi-algebraic functions, $E$ is a semi-algebraic set. Note that $(x, y) \in c l(W)$ if and only if $f(x, y)=0$; and $(x, y) \in \operatorname{vcl}_{\mathbb{R}^{n}}(W)$ if and only if $g(x, y)=0$. Thus, $(x, y) \in c l(W) \backslash v c l_{\mathbb{R}^{n}}(W)$ implies $x \in E$. Therefore, for all $x \in \mathbb{R}^{n} \backslash E,\left\{y \in \mathbb{R}^{m}:(x, y) \in \operatorname{cl}(W)\right\}=\left\{y \in \mathbb{R}^{m}:(x, y) \in v c l \mathbb{R}^{n}(W)\right\}$. Suppose $E$ is not lower-dimensional in $\mathbb{R}^{n}$. Then there is a semi-algebraic open set $\mathcal{O} \subseteq \mathbb{R}^{n}$ and an $\varepsilon>0$ with the property that for any $x \in \mathcal{O}$ there exists $(x, y) \in W$ such that $f(x, y)=0$ and $g(x, y) \geq \varepsilon$. The set

$$
G=\left\{(x, y) \in \mathcal{O} \times \mathbb{R}^{m}: f(x, y)=0 \text { and } g(x, y) \geq \varepsilon\right\}
$$

is semi-algebraic and its projection onto $\mathbb{R}^{n}$ is all of $\mathcal{O}$. So we can choose a semi-algebraic selection $\beta: \mathcal{O} \rightarrow \mathbb{R}^{m}$ with the property that $(x, \beta(x)) \in G$. By BZ94's (p.786) Lemma, there exists a semialgebraic open set $\mathcal{O}^{\prime} \subset \mathcal{O}$ on which $\beta$ is continuous. Since $(x, \beta(x)) \in \operatorname{cl}(W)$ for any $w \in \mathcal{O}^{\prime}$, there is a sequence $\left\{\left(x^{t}, y^{t}\right)\right\}_{t=1}^{\infty}$ in $W$ with limit $(x, \beta(x))$. From the continuity of $\beta,\left\|\beta\left(x^{t}\right)-\beta(x)\right\| \rightarrow 0$. Thus for $t$ large enough, $g\left(x^{t}, \beta\left(x^{t}\right)\right)<\varepsilon$, which contradicts the construction of $\beta$. Therefore, $E$ is a lower-dimensional in $\mathbb{R}^{n}$ and the result follows.

Lemma 3. Consider an extensive form $\Gamma$ with perfect recall. For each player $i$, there is a closed, lowerdimensional semi-algebraic subset $U_{i}^{0} \subset U_{i}$ such that, for any $Y_{-i} \subseteq \mathbb{Y}_{-i}, P B_{i}\left(Y_{-i}, u_{i}\right)=S B_{i}\left(Y_{-i}, u_{i}\right)$

\footnotetext{
${ }^{17}$ Chakrabarti and Topolyan (2016) adopted a similar backward approach to show the existence of sequential equilibrium. They backwardly constructed perturbations on strategies, while here we backwardly construct perturbations on payoffs.
} 
$\forall u_{i} \in U_{i} \backslash U_{i}^{0}$.

Proof: Let $Y_{-i} \subseteq \mathbb{Y}_{-i}$. By Lemma 1, SBi $\left(Y_{-i}, u_{i}\right)=\left\{y_{i} \in \mathbb{Y}_{i}:\left(u_{i}, x, y_{i}\right) \in \operatorname{cl}\left(\mathcal{R}_{i}\right) \cap Q\left(Y_{-i}\right)\right\}$ and $P B_{i}\left(Y_{-i}, u_{i}\right)=\left\{y_{i} \in \mathbb{Y}_{i}:\left(u_{i}, x, y_{i}\right) \in \operatorname{vcl}_{U_{i}}\left(\mathcal{R}_{i}\right) \cap Q\left(Y_{-i}\right)\right\}$. By the Tarski-Seidenberg Theorem, $\mathcal{R}_{i}$ is semi-algebraic. By Lemma $2, \operatorname{cl}\left(\mathcal{R}_{i}\right)$ and $v_{c l} l_{U_{i}}\left(\mathcal{R}_{i}\right)$ are generically equivalent on $U_{i}$ and hence $c l\left(\mathcal{R}_{i}\right) \cap Q\left(Y_{-i}\right)$ and $v c l_{U_{i}}\left(\mathcal{R}_{i}\right) \cap Q\left(Y_{-i}\right)$ are generically equivalent on $U_{i}$. Therefore, there is a closed, lower-dimensional semi-algebraic subset $U_{i}^{0} \subset U_{i}$ such that $P B_{i}\left(Y_{-i}, u_{i}\right)=S B_{i}\left(Y_{-i}, u_{i}\right) \forall u_{i} \in U_{i} \backslash U_{i}^{0}$. 
Lemma 4. Let $F: U \rightrightarrows \mathbb{R}^{n}$ and $F^{\prime}: U \rightrightarrows \mathbb{R}^{n}$. Suppose $V^{0} \equiv\left\{u^{0} \in U: F\left(u^{0}\right) \neq F^{\prime}\left(u^{0}\right)\right\}$ is a lower dimensional subset of $U$. Then $F(u) \subseteq F^{\prime}(u)$ for all $u \in U$ at which $F(\cdot)$ is lower hemi-continuous and $F^{\prime}(\cdot)$ is upper hemi-continuous.

Proof of Lemma 4. Since $V^{0}$ is lower-dimensional, $V^{0}$ contains no open set in $U$. Let $u \in U$. Therefore, we can find a sequence $\left\{u^{t}\right\}_{t=1}^{\infty}$ in $U \backslash V^{0}$ such that $u^{t} \rightarrow u$ and $F\left(u^{t}\right)=F^{\prime}\left(u^{t}\right)$ for all $t$. If $y \in F(u)$, by lower hemi-continuity of $F(\cdot)$, there exists a subsequence $u^{t_{k}} \rightarrow u$ such that $y^{k} \rightarrow y$ and $y^{k} \in F\left(u^{t_{k}}\right)=F^{\prime}\left(u^{t_{k}}\right)$. Since the correspondence $F^{\prime}(\cdot)$ is upper hemi-continuous, $y \in F^{\prime}(u)$. That is, $F(u) \subseteq F^{\prime}(u)$.

Proof of Theorem 1: By Lemma 3, for each player $i \in N$, there is a closed, lower-dimensional semi-algebraic subset $U_{i}^{0} \subset U_{i}=\mathbb{R}^{|Z|}$ such that for all $u_{i} \in U_{i} \backslash U_{i}^{0}, P B_{i}\left(Y_{-i}, u_{i}\right)=S B_{i}\left(Y_{-i}, u_{i}\right)$ for all $Y_{-i} \subseteq \mathbb{Y}_{-i}$; hence, $P B_{i}\left(\Pi_{j \neq i} c o^{e}\left(Y_{j}\right), u_{i}\right)=S B_{i}\left(\Pi_{j \neq i} c o^{e}\left(Y_{j}\right), u_{i}\right)$ for all $\Pi_{j \neq i} Y_{j} \subseteq \mathbb{Y}_{-i}$. Therefore, for all the payoffs $u \in U \backslash U^{0}$ where $U^{0}=\cup_{i \in N}\left(U_{i}^{0} \times U_{-i}\right)$, a course of action $\sigma$ is perfectly supported by $Y$ in $\Gamma(u)$ if and only if $\sigma$ is sequentially supported by $Y$ in $\Gamma(u)$. Thus, we find a closed, lower-dimensional semi-algebraic subset $U^{0} \subset U$ such that $\Sigma^{\text {sequential }}(u)=\Sigma^{\text {perfect }}(u)$ and $\top_{\sigma}^{\text {sequential }}(u)=\top_{\sigma}^{\text {perfect }}(u)$ for all $u \in U \backslash U^{0}$ and for an arbitrary given course of action $\sigma$ in $\Gamma$.

Because $P B_{i}\left(\Pi_{j \neq i} c o^{e}\left(Y_{j}\right), u_{i}\right)=S B_{i}\left(\Pi_{j \neq i} c o^{e}\left(Y_{j}\right), u_{i}\right)$ for all $i \in N$ and $u \in U \backslash U^{0},\left\{u^{0} \in U\right.$ : $\left.\Upsilon^{\text {sequential }}\left(u^{0}\right) \neq \Upsilon^{\text {perfect }}\left(u^{0}\right)\right\} \subseteq U^{0}$ is a lower dimensional subset of $U$. By Lemma $4, \Upsilon^{\text {sequential }}(u) \subseteq$ $\Upsilon^{\text {perfect }}(u)$ for all the payoffs $u \in U$ at which $\Upsilon^{\text {sequential }}(\cdot)$ is lower hemi-continuous and $\Upsilon^{\text {perfect }}(\cdot)$ is upper hemi-continuous. But, since any perfect-MACA in $\Gamma(u)$ is also a sequential-MACA in $\Gamma(u)$, $\Upsilon^{\text {perfect }}(u) \subseteq \Upsilon^{\text {sequential }}(u)$ for all $u \in U$. Hence, $\Upsilon^{\text {sequential }}(u)=\Upsilon^{\text {perfect }}(u)$ for all the payoffs $u \in U$ at which $\Upsilon^{\text {sequential }}(\cdot)$ is lower hemi-continuous and $\Upsilon^{\text {perfect }}(\cdot)$ is upper hemi-continuous. Similarly, for an arbitrary given course of action $\sigma$ in $\Gamma, \top_{\sigma}^{\text {sequential }}(u)=\top_{\sigma}^{\text {perfect }}(u)$ for all the payoffs $u \in U$ at which $\top_{\sigma}^{\text {sequential }}(\cdot)$ is lower hemi-continuous and $\top_{\sigma}^{\text {perfect }}(\cdot)$ is upper hemi-continuous.

\section{Proof of Corollary 1: Define}

$$
\stackrel{o}{\mathcal{R}}=\left\{(u, x, y) \in U \times \mathbb{Y} \times \mathbb{Y}:\left(u_{i}, x, y_{i}\right) \in \mathcal{R}_{i} \forall i\right\}
$$

that is, $\stackrel{\circ}{\mathcal{R}}$ is the set of "joint" perfectly rational states in which the players have consistently aligned beliefs about the opponent players' behavior. Clearly, $(u, x, y) \in v l_{U}(\stackrel{o}{\mathcal{R}})$ iff there exists a common sequence $x^{t} \rightsquigarrow x$ such that for each player $i, y_{i}$ is the best response along the trembling sequence $x^{t}$. By Definition $\mathrm{A} 1(\mathrm{i}),(u, y, y) \in v c l_{U}(\stackrel{\circ}{\mathcal{R}})$ iff $y$ is a perfect equilibrium; by Kreps and Wilson's (1982) Proposition 6, $(u, y, y) \in \operatorname{cl}(\stackrel{o}{\mathcal{R}})$ iff $y$ is a sequential equilibrium strategy profile. By the Tarski-Seidenberg Theorem, $\stackrel{o}{\mathcal{R}}$ is semi-algebraic. By Lemma $2, \operatorname{cl}(\stackrel{o}{\mathcal{R}})$ and $v l_{U}(\stackrel{o}{\mathcal{R}})$ are generically equivalent. Consequently, there is a closed, lower-dimensional semi-algebraic subset $U^{0} \subset U$ such that 
for all $u \in U \backslash U^{0}$, any sequential equilibrium strategy profile is a perfect equilibrium in game $\Gamma(u)$.

Now, suppose that the sequential equilibrium correspondence $S E(\cdot)$ is lower hemi-continuous and the perfect equilibrium correspondence $P E(\cdot)$ is upper hemi-continuous at $u \in U$. Since $\left\{u^{0} \in U: S E\left(u^{0}\right) \neq P E\left(u^{0}\right.\right.$ $U^{0}$ is a lower dimensional subset, by Lemma $4, S E(u) \subseteq P E(u)$. Thus, $S E(u)=P E(u)$.

Proof of Corollary 2: (i) By Definition A1(iii)-(iv), a strategy profile $y \in \mathbb{Y}$ is a weakly perfect (or weakly sequential) equilibrium in $\Gamma(u)$ iff for each player $i, y_{i} \in P B_{i}\left(y_{-i}, u_{i}\right)=P B_{i}\left(\Pi_{j \neq i} c o^{e}\left(y_{j}\right), u_{i}\right)$ (or $y_{i} \in S B_{i}\left(y_{-i}, u_{i}\right)=S B_{i}\left(\Pi_{j \neq i} c o^{e}\left(y_{j}\right), u_{i}\right)$ ). By Definition 2, the strategy profile $y$ is a weakly perfect (or weakly sequential) equilibrium in $\Gamma(u)$ iff $y$ is a perfect (or sequential) MACA in $\Gamma(u)$. By Theorem 1, for generic $u \in U \backslash U^{0}$, the set of weakly sequential equilibria in $\Gamma(u)$ coincides with the set of weakly perfect equilibria in $\Gamma(u)$.

(ii) By Definition A2, a product set $Y=\prod_{i \in N} Y_{j} \subseteq \mathbb{Y}$ is a perfectly (or sequentially) rationalizable set in $\Gamma(u)$ iff for each player $i, Y_{i} \subseteq P B_{i}\left(\Pi_{j \neq i} c o^{e}\left(Y_{j}\right), u_{i}\right)$ (or $\left.Y_{i} \subseteq S B_{i}\left(\Pi_{j \neq i} c o^{e}\left(Y_{j}\right), u_{i}\right)\right)$. By Definition 2 , the set $Y$ is a perfectly (or sequentially) rationalizable set in $\Gamma(u)$ iff $Y$ perfectly (or sequentially) supports the null MACA in $\Gamma(u)$. By Theorem 1, for generic payoffs $u \in U \backslash U^{0}$, the union of sequentially rationalizable sets in $\Gamma(u)$ coincides with the union of perfectly rationalizable sets in $\Gamma(u)$; that is, the set of sequentially rationalizable strategy profiles in $\Gamma(u)$ coincides with the set of perfectly rationalizable strategy profiles in $\Gamma(u)$.

(iii) This result follows directly from the following lemma:

Lemma 5. A path resulting from an SRSCE in Definition A3 is a path sequential-MACA in Definition 2 and vice versa.

Proof of Lemma 5: " $\Rightarrow$ " Suppose $\widehat{y}$ is an SRSCE in $\Gamma(u)$. By Definition A3, there exists $Y=$ $\Pi_{i \in N} Y_{i} \subseteq \mathbb{Y}$, for each $i \in N$ and each $y_{i} \in Y_{i}$, we can find $y_{-i} \in \Pi_{j \neq i} c o\left(Y_{j}\right)$ such that $y_{h}=\widehat{y}_{h}$ if $h \in H$ is reachable under $\widehat{y}$ and $y_{i} \in S B_{i}\left(y_{-i}, u_{i}\right)$. Construct a path course of action $\sigma$ (associated with $\widehat{y}$ ) as follows: For any $h \in H$,

$$
\sigma_{h}=\left\{\begin{array}{ll}
\widehat{y}_{h}, & \text { if } h \text { is reachable under } \widehat{y} \\
\emptyset, & \text { otherwise }
\end{array} .\right.
$$

By the construction of $\sigma$, if $\sigma_{h} \neq \emptyset$, then $y_{h}=\sigma_{h}$ for all $y \in Y$. Moreover, since $y_{i} \in S B_{i}\left(y_{-i}, u_{i}\right)$ and $y_{-i} \in \Pi_{j \neq i} c o^{e}\left(Y_{j}\right), y_{i} \in S B_{i}\left(\Pi_{j \neq i} c o^{e}\left(Y_{j}\right), u_{i}\right)$. By Definition 2, the path course of action $\sigma$ (associated with $\widehat{y})$ is a path sequential-MACA in $\Gamma(u)$ supported by $Y$.

" $\Leftarrow$ " Suppose $\sigma$ is a path sequential-MACA in $\Gamma(u)$ supported by $Y=\Pi_{i \in N} Y_{i} \subseteq \mathbb{Y}$. By Definition 2, for each player $i$ and each $\widehat{y}_{i} \in Y_{i}$, there exists $\widehat{y}_{-i} \in \Pi_{j \neq i} c o^{e}\left(Y_{j}\right)$ such that $\widehat{y}_{i} \in S B_{i}\left(\widehat{y}_{-i}, u_{i}\right)$; moreover, if $\sigma_{h} \neq \emptyset, y_{h}=\sigma_{h}$ for all $y \in Y$ and hence $\widehat{y}_{h}=\sigma_{h}$. That is, $\widehat{y}_{h}=\sigma_{h}$ if $h$ is reachable under $\widehat{y}$. By Definition A3, $\widehat{y}$ is an SRSCE that results in the same path $\sigma$.

To show the last part of Corollary 2, let $\sigma$ be a course of action in $\Gamma$. Notice that the player-by- 
player union of the sequentially/perfectly $\sigma$-supporting sets in $\Gamma(u)$ is again a sequentially/perfectly $\sigma$-supporting set in $\Gamma(u)$. Thus, $\Upsilon_{\sigma}^{\text {sequential }}(u)$ is the largest sequentially $\sigma$-supporting set and $\Upsilon_{\sigma}^{\text {perfect }}(u)$ is the largest perfectly $\sigma$-supporting set. By Theorem 1, the largest sequentially $\sigma$-supporting set coincides with the largest perfectly $\sigma$-supporting set for generic payoffs $u \in U \backslash U^{0}$.

Proof of Corollary 3: Consider a finite normal form $\Gamma=\left(N,\left\{A_{i}\right\}_{i \in N}\right)$. Let $\left(W^{k}(u)\right)_{k=0}^{K}$ be an IEWDS procedure in $\Gamma(u)$, where $W^{k}(u)=\Pi_{i \in N} W_{i}^{k}(u)$ such that $W_{i}^{0}(u)=A_{i}$ and $W_{i}^{k}(u) \subseteq A_{i}$ includes the set of $i$ 's surviving weakly undominated actions in the $(k-1)$-th round of elimination for all $k \geq 1$. Let $i \in N$ and $k=1,2, \ldots, K$. Because $\Gamma$ is a normal form, by Pearce's (1984) Lemma $3, a_{i} \in W_{i}^{k}(u)$ is not strictly dominated in $W^{k}(u)$ iff $a_{i} \in S B_{i}\left(\Delta\left(W_{-i}^{k}(u)\right), u_{i}\right)$; by Pearce's $(1984)$ Lemma $4, a_{i} \in W_{i}^{k}(u)$ is not weakly dominated in $W^{k}(u)$ iff $a_{i} \in P B_{i}\left(\Delta\left(W_{-i}^{k}(u)\right), u_{i}\right)$. By Lemma 3 , there is a closed, lower-dimensional subset $U_{i}^{0} \subset U_{i}$ such that for all $u_{i} \in U_{i} \backslash U_{i}^{0}, S B_{i}\left(Y_{-i}, u_{i}\right)=$ $P B_{i}\left(Y_{-i}, u_{i}\right) \forall Y_{-i} \subseteq \Delta\left(A_{-i}\right)$. Letting $Y_{-i}=\left\{y_{-i} \in \Delta\left(A_{-i}\right): y_{-i}\right.$ has full support on $\left.W_{-i}^{k}(u)\right\}$, we have $S B_{i}\left(\Delta\left(W_{-i}^{k}(u)\right), u_{i}\right)=P B_{i}\left(\Delta\left(W_{-i}^{k}(u)\right), u_{i}\right)$ for all $u_{i} \in U_{i} \backslash U_{i}^{0}$. Define $U^{0}=\cup_{i \in N}\left(U_{i}^{0} \times U_{-i}\right)$. Therefore, $U^{0} \subset U$ is a closed, lower-dimensional semi-algebraic subset such that for all $u \in U \backslash U^{0}$, $a \in W^{k}(u) \backslash W^{k+1}(u)$ iff $a \in W^{k}(u)$ is strictly dominated in $W^{k}(u)$ and hence $\left(W^{k}(u)\right)_{k=0}^{K}$ is an IESDS procedure in $\Gamma(u)$. Since IESDS is order-independent in finite games, IEWDS is generically an orderindependent procedure.

\section{References}

1. Aryal, G., Stauber, R.: Trembles in extensive games with ambiguity averse players. Econ. Theory 57, 1-40 (2014)

2. Asheim, G.B., Perea, A.: Sequential and quasi-perfect rationalizability in extensive games. Games Econ. Behav. $53,15-42(2005)$

3. Aumann, R.J.: What is game theory trying to accomplish?. In: K. Arrow and S. Honkapohja (eds.), Frontiers of Economics, pp.28-76, Basil Blackwell, Oxford (1985)

4. Battigalli, P.: On rationalizability in extensive form games. J. Econ. Theory 74, 40-61 (1997)

5. Battigalli, P., Cerreia-Vioglio, S., Maccheroni, F., Marinacci, M.: Self-confirming equilibrium and model uncertainty. Am. Econ. Rev. 105, 646-77 (2015)

6. Bernheim, B.D.: Rationalizable strategic behavior. Econometrica 52, 1007-1028 (1984)

7. Blume, L.E., Zame, W.R.: The algebraic geometry of competitive equilibrium. In: W. Neuefeind and R.G. Riezman (eds), Essays in General Equilibrium and International Trade: In Memoriam Trout Rader, pp.53-66, Springer-Verlag, Berlin (1993)

8. Blume, L.E., Zame, W.R.: The algebraic geometry of perfect and sequential equilibrium. Econometrica 62 , 783-794 (1994)

9. Chakrabarti, S.K., Topolyan, I.: An extensive form-based proof of the existence of sequential equilibrium. Econ. Theory Bull. 4, 355-365 (2016) 
10. J. Bochnak, J., Coste, M., Roy, M.-F.: Geometie Algebrique Reelle, Ergebnisse der Mathematik und ihrer Grenzgebiete. Springer-Verlag, Berlin (1987)

11. Börgers, T.: Weak dominance and approximate common knowledge. J. Econ. Theory 64, 265-276 (1994)

12. Brandenburger, A., Friedenberg, A.: The relationship between rationality on the matrix and the tree. NYU Working paper (2003)

13. Demichelis, S., Ritzberger, K.: From evolutionary to strategic stability. J. Econ. Theory 113, 51-75 (2003)

14. Dekel, E., Fudenberg, D., Levine, D.K.: Payoff information and self-confirming equilibrium. J. Econ. Theory 89, 165-185 (1999)

15. Dekel, E., Fudenberg, D., Levine, D.K.: Subjective uncertainty over behavior strategies: A correction. J. Econ. Theory 104, 473-478 (2002)

16. Esponda, I.: Rationalizable conjectural equilibrium: a framework for robust predictions. Theoretical Econ. 8, 467-501 (2013)

17. Fudenberg, D., Kamada, Y.: Rationalizable partition-confirmed equilibrium. Theoretical Econ. 3, 775-806 (2015)

18. Fudenberg, D., Levine, D.K.: Self-confirming equilibrium. Econometrica 61, 523-545 (1993)

19. Fudenberg, D., Levine, D.K.: Superstition and rational learning. Am. Econ. Rev. 96, 630-651 (2006)

20. Fudenberg, D., Levine, D.K.: Self-confirming equilibrium and the Lucas critique, J. Econ. Theory 144, 2354-2371 (2009)

21. Fudenberg, D. and Tirole, J.: Game Theory. MIT Press, Cambridge, MA (1991)

22. Govindan, S., McLennan, A.: On the generic finiteness of equilibrium outcome distributions in game forms. Econometrica 69, 455-471 (2001)

23. Govindan, S., Wilson, R.: Direct proofs of generic finiteness of Nash equilibrium outcomes. Econometrica 69, 765-769 (2001)

24. Govindan, S., Wilson, R.: Sufficient conditions for stable equilibria. Theoretical Econ. 1, 167-206 (2006)

25. Govindan, S., Wilson, R.: Axiomatic equilibrium selection for generic two-player games. Econometrica 80, 1639-1699 (2012)

26. Greenberg, J., Gupta, S., Luo, X.: Mutually acceptable courses of action. Econ. Theory 40, 91-112 (2009)

27. Haller, H., Lagunoff, R.: Genericity and Markovian behavior in stochastic games. Econometrica 80, 1639-1699 (2012)

28. Halpern, J.Y.: A nonstandard characterization of sequential equilibrium, perfect equilibrium, and proper equilibrium. Int. J. Game Theory 38, 37-49 (2009)

29. Hardt, R.M.: Semi-algebraic local-triviality in semi-algebraic mappings. American J. Math. 102, 291-302 (1980)

30. Herings, P.J.J., Vannetelbosch, V.J.: Refinements of rationalizability for normal-form games. Int. J. Game Theory 28, 53-68 (1999) 
31. Hillas, J., Kohlberg, E.: Foundations of strategic equilibrium. In: R.J. Aumann and S. Hart (eds.), Handbook of Game Theory with Economic Applications, Vol. 3, pp.1597-1663, North-Holland, Amsterdam (2002)

32. Kohlberg, E., Mertens, J.-F.: On the strategic stability of equilibria. Econometrica 54, 1003-1039 (1986)

33. Kohlberg, E., Reny, P.J.: Independence on relative probability spaces and consistent assessments in game trees. J. Econ. Theory 75, 280-313 (1997)

34. Kreps, D., Wilson, R.: Sequential equilibria. Econometrica 50, 1003-1038 (1982)

35. Kubler, K., Schmedders, K.: Competitive equilibria in semi-algebraic economies. J. Econ. Theory 145, 301-330 (2010)

36. Luo, X., Wang, B.: An epistemic characterization of MACA. NUS Working paper (2019)

37. McKelvey R.D., McLennan, A.: Computation of equilibria in finite games. In: H.M. Amman, D.A. Kendrick and J. Rust (eds.), Handbook of Computational Economics, Vol. 1, pp.87-142, North-Holland, Amsterdam (1996)

38. Myerson, R.B.: Refinements of the Nash equilibrium concept. Int. J. Game Theory 15, 133-154 (1978)

39. Osborne, M.J., Rubinstein, A.: A Course in Game Theory. MIT Press, Cambridge, MA (1994)

40. Pearce, D.: Rationalizable strategic behavior and the problem of perfection. Econometrica 52, 1029-1051 (1984)

41. Perea, A.: A note on the one-deviation property in extensive form games. Games Econ. Behav. 40, 322-338 (2002)

42. Perea, A.: Why forward induction leads to the backward induction outcome: A new proof for Battigalli's theorem. Games Econ. Behav. 110, 120-138 (2018)

43. Perea, A., Jansen, M., Peters, H.: Characterization of consistent assessments in extensive form games. Games Econ. Behav. 21, 238-252 (1997)

44. Pimienta, C., Litan, C.: Conditions for equivalence between sequentiality and subgame perfection. Econ. Theory $35,539-553(2008)$

45. Pimienta, C., Shen, J.: On the equivalence between (quasi-) perfect and sequential equilibria. Int. J. Game Theory 43, 395-402 (2014)

46. Ritzberger, K.: The theory of normal form games from the differentiable viewpoint. Int. J. Game Theory 23, 207-236 (1994)

47. Rubinstein, A., Wolinsky, A.: Rationalizable conjectural equilibrium: Between Nash and rationalizability. Games Econ. Behav. 6, 299-311 (1994)

48. Selten, R.: Reexamination of the perfectness concept for equilibrium points in extensive games. Int. J. Game Theory 4, 25-55 (1975)

49. Shimoji, M.: On the equivalence of weak dominance and sequential best response. Games Econ. Behav. 48, 385-402 (2004)

50. van Damme, E.: Stability and Perfection of Nash Equilibria. Springer-Verlag, Berlin (1992)

51. van Damme, E.: On the state of the art in game theory: An interview with Robert Aumann. Games Econ. Behav. 24, 181-210 (1998)

52. Voorneveld, M.: Persistent retracts and preparation. Games Econ. Behav. 51, 228-232 (2005) 